\title{
String Amplitudes from Field-Theory Amplitudes and Vice Versa
}

\author{
Song He, ${ }^{1,2, *}$ Fei Teng, ${ }^{3, \dagger}$ and Yong Zhang ${ }^{1,2, \$}$ \\ ${ }^{1}$ CAS Key Laboratory of Theoretical Physics, Institute of Theoretical Physics, Chinese Academy of Sciences, Beijing 100190, China \\ ${ }^{2}$ School of Physical Sciences, University of Chinese Academy of Sciences, No.19A Yuquan Road, Beijing 100049, China \\ ${ }^{3}$ Department of Physics and Astronomy, Uppsala University, 75108 Uppsala, Sweden
}

(Received 22 February 2019; published 30 May 2019)

\begin{abstract}
We present an integration-by-parts reduction of any massless tree-level string correlator to an equivalence class of logarithmic functions, which can be used to define a field-theory amplitude via a Cachazo-He-Yuan (CHY) formula. The string amplitude is then shown to be the double copy of the fieldtheory one and a special disk or sphere integral. The construction is generic as it applies to any correlator that is a rational function of correct SL(2) weight. By applying the reduction to open bosonic or heterotic strings, we get a closed-form CHY integrand for the $(D F)^{2}+$ Yang-Mills $+\phi^{3}$ theory.
\end{abstract}

DOI: $10.1103 /$ PhysRevLett.122.211603

Introduction.-Recent years have seen enormous advances in understanding structures of scattering amplitudes in quantum field theories (c.f. [1-3]), and many crucial insights have originated from string theory. The field-theory limit of the celebrated Kawai-Lewellen-Tye (KLT) relations [4] between tree amplitudes in open- and closed-string theory gives double copy relations linking gauge-theory amplitudes to gravitational ones [5]. Based on a remarkable duality between color and kinematics due to Bern, Carrasco, and Johansson (BCJ) [6], double copy has been extended to quantum level and become the state-ofthe-art method for multiloop calculations in supergravities [7]. Apart from the original KLT relations, string theory has provided constructions of amplitude representation that respects color-kinematics duality at tree and loop level [8-12], and amplitude relations in gauge theory, i.e., BCJ relations $[13,14]$, and beyond $[15,16]$.

More surprisingly, it has been realized that tree-level open- and closed-superstring amplitudes themselves can be obtained via a double copy [17]. By rearranging the worldsheet correlator with massless states in type-I theory $[9,10]$, we can decompose disk amplitudes into field-theory KLT products of super-Yang-Mills (SYM) amplitudes and universal basis of disk integrals called $Z$ integrals. The former contain the full polarization information but no nontrivial $\alpha^{\prime}$ dependence, while the latter encode $\alpha^{\prime}$ expansion and are interpreted as amplitudes in an effective field theory dubbed $Z$ theory [18-20]. Very recently, this has been generalized to bosonic and heterotic strings

Published by the American Physical Society under the terms of the Creative Commons Attribution 4.0 International license. Further distribution of this work must maintain attribution to the author(s) and the published article's title, journal citation, and DOI. Funded by SCOAP ${ }^{3}$.
[21,22]: now the field-theory amplitudes also contain tachyon poles, and they were conjectured to come from the $(D F)^{2}+\mathrm{YM}+\phi^{3}$ Lagrangian, with $\alpha^{\prime}$ related to its mass parameter [23]. With $Z$ integrals replaced by certain sphere integrals, we can get closed-string amplitudes via a double copy from these field theories [24].

An alternative framework that manifests and extends the double copy in field theories is the Cachazo-He-Yuan (CHY) formulation [25,26], which expresses tree amplitudes in a large class of massless theories based on scattering equations [27]. Together with loop-level generalizations [28-32], these have led to new double-copy realization of various theories [33], and one-loop extensions of KLT and amplitude relations [34,35]. It is now well established that tree and loop CHY formulas can be derived from worldsheet models known as ambitwistor string theory [36,37], where CHY integrand is given by correlators therein. Despite significant progress [38-42], it is fair to say that the origin of ambitwistor strings and the relations to strings at finite $\alpha^{\prime}$ remain mysterious. A related outstanding question is how such double copies for strings can be understood via ambitwistor strings, especially for bosonic and heterotic strings which would require $\mathrm{CHY}$ formulas with nontrivial $\alpha^{\prime}$ dependence.

In this Letter, we present a double-copy construction for any massless string amplitude in terms of field-theory amplitudes defined by a CHY formula, where the integrand can be directly obtained from the original string correlator. We show algorithmically how to reduce a completely general rational function with correct SL(2) weight (which we refer to as a string correlator), via integration by parts (IBP) to an equivalent class of logarithmic functions, and any of them serves as a CHY half-integrand that gives desired field-theory amplitudes. Once the logarithmic class is reached, the next step is to use scattering equations (SE) to obtain equivalent half-integrands, which are no longer 
logarithmic but usually take a more compact form and make some properties more manifest. The same holds for closed-string correlators where we reduce the holomorphic part by IBP.

As the main application illustrating the power of this method, we perform IBP for all scalar-gluon correlators of compactified open bosonic strings, or equivalently the holomorphic part in heterotic strings, to produce logarithmic functions as $\mathrm{CHY}$ half-integrands. By using SE, we further present a remarkably simple formula for the halfintegrand to any multiplicities which gives amplitudes for the single-trace sector of the $(D F)^{2}+\mathrm{YM}+\phi^{3}$ theory. The formula for multitrace amplitudes and pure gluon ones are contained in our result as well; thus it gives CHY representations for all tree amplitudes in this theory.

CHY integrand from string correlator.-The double copy construction at tree level can be most conveniently expressed by KLT product of color-ordered amplitudes. $\mathcal{M}_{C}=\mathcal{M}_{A} \otimes \mathcal{M}_{B}$ is defined as

$$
\mathcal{M}_{C}=\sum_{\alpha, \beta \in S_{n-3}} \mathcal{M}_{A}(\alpha) S[\alpha \mid \beta] \mathcal{M}_{B}(\beta),
$$

where the $(n-3)$ ! dimensional matrix $S[\alpha \mid \beta]$ is the socalled momentum kernel [43] for orderings $\alpha, \beta$ in a minimal basis. Instead of writing $S$ explicitly, it suffices to say that it is the inverse of the matrix formed by doublepartial amplitudes in biadjoint $\phi^{3}$ theory: $S=m^{-1}$ [26], for any choice of basis such that $m$ is invertible.

A generic massless open-string tree amplitude is given by a disk integral with $\rho \in S_{n} / Z_{n}$ denoting the ordering for integration domain $z_{\rho(i)}<z_{\rho(i+1)}$

$$
\mathcal{M}_{n}^{\text {string }}(\rho)=\int_{\rho} \underbrace{\frac{d^{n} z}{\operatorname{volSL}(2, \mathbb{R})}}_{:=d \mu_{n}^{\text {string }}} \overbrace{i<j}^{:=\mathrm{KN}}\left|z_{i j}\right|^{s_{i j}} \mathcal{I}_{n}(z),
$$

where $s_{i j}:=\alpha^{\prime} k_{i} \cdot k_{j}, \quad z_{i j}:=z_{i}-z_{j}$; one can fix three punctures, e.g., $\left(z_{1}, z_{n-1}, z_{n}\right)=(0,1, \infty)$, using $\operatorname{SL}(2, \mathbb{R})$ redundancy, and the product in the Koba-Nielsen factor is over $1 \leq i<j \leq n-1$ with this fixing. After stripping it off, the (reduced) string correlator $\mathcal{I}_{n}$ is a rational function of $z \mathrm{~s}$ which depends on details of vertex operators. The only requirement here is that it has the correct $\mathrm{SL}(2)$ weight: with $z_{a} \rightarrow\left(\alpha+\beta z_{a}\right) /\left(\gamma+\delta z_{a}\right)$ and $\alpha \delta-\beta \gamma=1$, it must transform as $\mathcal{I}_{n} \rightarrow \prod_{a=1}^{n}\left(\gamma+\delta z_{a}\right)^{2} \mathcal{I}_{n}$.

General claim: Our general claim, as summarized in Fig. 1, is that $\mathcal{M}_{n}^{\text {string }}(\rho)$ is the double copy of $Z$ integrals [disk integral with a Parke-Taylor (PT) factor [17] ]

$$
Z_{\rho}(\pi)=\int_{\rho} \frac{d \mu_{n}^{\text {string }}}{z_{\pi_{1} \pi_{2}} z_{\pi_{2} \pi_{3}} \cdots z_{\pi_{n} \pi_{1}}}:=\int_{\rho} d \mu_{n}^{\text {string }} \mathrm{PT}(\pi),
$$

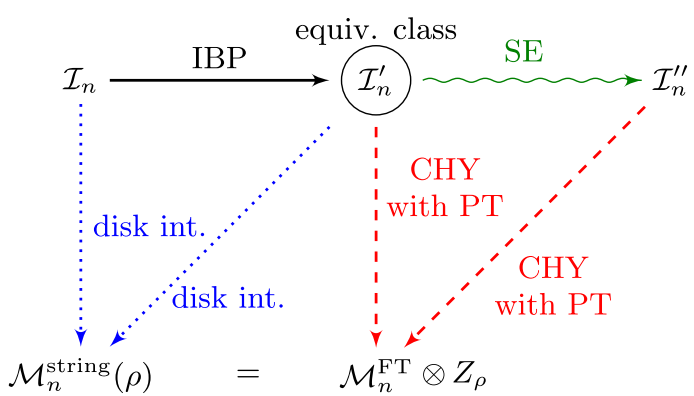

FIG. 1. Double copy for string amplitudes, and CHY halfintegrands from string correlator via IBP and SE.

with color-ordered amplitudes in a field theory. The latter is defined by a CHY formula, with integrands given by a PT factor as defined above, and a logarithmic function $\mathcal{I}_{n}^{\prime}$ obtained from an IBP reduction of $\mathcal{I}_{n}$

$$
\mathcal{M}_{n}^{\mathrm{FT}}(\rho):=\int \underbrace{\frac{d^{n} z}{\operatorname{volSL}(2, \mathbb{C})} \prod_{i}^{\prime} \delta\left(\sum_{j \neq i} \frac{s_{i j}}{z_{i j}}\right)}_{:=d \mu_{n}^{\mathrm{CHY}}} \operatorname{PT}(\rho) \mathcal{I}_{n}^{\prime}(z) .
$$

Here the integrals are localized by the $n-3$ delta functions imposing scattering equations [25,27]. Logarithmic functions are defined to have only logarithmic singularities on boundaries of the moduli space of $n$-punctured Riemann spheres; equivalently, such a function can be written as a linear combination of PT factors [44-46].

The idea that string amplitude can be written as a double copy has been realized in various theories $[9,10,21,22]$ and expected to hold for generic $\mathcal{I}_{n}$. For example, by using intersection theory, we can obtain $\mathcal{I}_{n}^{\prime}$ from $\mathcal{I}_{n}$ by computing intersection numbers [47]. In this Letter, we will give a concrete and streamlined IBP algorithm that reduces a generic $\mathcal{I}_{n}$ explicitly to a class of logarithmic functions; the algorithm lands $\mathcal{I}_{n}^{\prime}$ on a nonminimal basis of PT factors, making further simplifications accessible.

We actually have an equivalence class of logarithmic functions denoted as $\mathcal{I}_{n}^{\prime} \stackrel{\mathrm{IBP}}{\cong} \mathcal{I}_{n}$ : any $\mathcal{I}_{n}^{\prime}$ gives the same string integral as that of $\mathcal{I}_{n}$. As we will show shortly, $\mathcal{I}_{n}^{\prime}$ gives exactly $\mathcal{M}_{n}^{\mathrm{FT}}$ needed for the double copy, via CHY formula with a PT factor; any $\mathcal{I}_{n}^{\prime}$ in the class gives the same result since they are also in an equivalent class on the support of SE. While the explicit form of $\mathcal{I}_{n}^{\prime}$ gets more complicated as $n$ grows, we emphasize that it can usually be simplified greatly by use of SE (see Fig. 1). The manipulation from $\mathcal{I}_{n}^{\prime}$ to $\mathcal{I}_{n}^{\prime \prime}$ is in a sense the reversed one as going from $\mathcal{I}_{n}$ to $\mathcal{I}_{n}^{\prime}$ : while being nonlogarithmic, usually $\mathcal{I}_{n}^{\prime \prime}$ allows an allmultiplicity expression that makes other properties, such as gauge invariance, manifest. The primary example of this double copy is "type-I $=Z \otimes S Y M "[9,10]$, where the class of logarithmic functions from type-I correlator can be 
used to produce SYM amplitudes. It is now well known that the CHY half-integrand for external gluons, which takes a remarkably simple form $\mathcal{I}_{n}^{\prime \prime}=\mathrm{Pf}^{\prime} \boldsymbol{\Psi}_{n}$ [48], is equivalent to $\mathcal{I}_{n}^{\prime}$ on the support of SE.

Last, but not the least, one can generalize the double copy to closed-string amplitudes by replacing $Z$ integrals by certain sphere integrals. As conjectured in Refs. [24,49,50] and proven in Refs. [51,52], the latter can be obtained as the single-valued (sv) projection $[53,54]$ of open-string amplitudes, and e.g., we have "type-II = sv(type-I) $\otimes$ SYM.” All of our discussions hold for such double copies where IBP is applied to the holomorphic part of closed-string correlator $\mathcal{I}_{n}(z)$ (with the antiholomorphic part untouched): schematically we write [22] $\mathcal{M}_{n}^{\text {closed }}=$ $\mathcal{M}_{n}^{\mathrm{FT}} \otimes \operatorname{sv}\left(\mathcal{M}_{n}^{\text {open }}\right)$, with the $\mathrm{CHY}$ half-integrand for $\mathcal{M}_{n}^{\mathrm{FT}}$ obtained from the holomorphic correlator, $\mathcal{I}_{n}^{\prime} \stackrel{\mathrm{IBP}}{\cong} \mathcal{I}_{n}$.

Derivation: IBP reduction and double copy: We can conveniently represent any rational functions involving only $z_{i j}$ 's by a graph: we denote each $z_{i j}$ in the denominator (numerator) by a solid (dashed) line directed from node $i$ to $j$. With the gauge fixing, e.g., $z_{n} \rightarrow \infty$, all the labeled trees are logarithmic functions since we can write them as a sum of PT factors [55].

The derivation of our general claim consists of two steps. We first give a method for reducing any string correlator to logarithmic functions via IBP relations. Next, we show that such logarithmic functions are precisely the $\mathrm{CHY}$ integrands for those field theories that enter the stringamplitude double copy mentioned above.

IBP reduction: We start with a simple example $\mathcal{I}_{4}=$ $\left(1 / z_{12}^{2} z_{34}^{2}\right)$, which, after we gauge fix $\left(z_{1}, z_{3}, z_{4}\right)=(0,1, \infty)$ and subtract the total derivative $\partial_{z_{2}}\left[\left(z_{2}^{s-1}\left(1-z_{2}\right)^{t}\right) /(s-1)\right]$, reduces to a logarithmic function; in this case a PT factor after restoring the $\mathrm{SL}(2)$ covariance

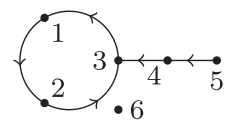

$\left(z_{7} \rightarrow \infty\right)$

(a) tadpole

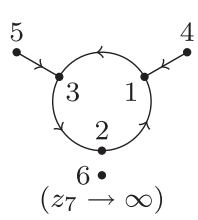

(b) multibranch

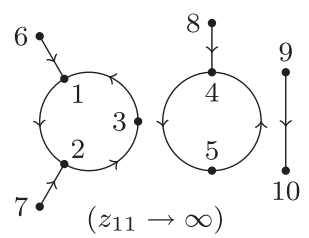

(c) generic
FIG. 2. Examples of rational functions of $z_{i j}$ with no numerators or connected subcycles after gauge fixing. For this case, gauge fixing always results in a single labeled tree.

$$
\frac{1}{z_{12}^{2} z_{34}^{2}} \stackrel{\operatorname{IBP}}{\cong} \frac{t}{1-s} \frac{1}{z_{12} z_{23} z_{34} z_{41}}=\frac{t}{1-s} \mathrm{PT}(1234),
$$

where $s=s_{12}$ and $t=s_{23}$. This example represents the simplest scenario where only a single subcycle and a labeled line are left after gauge fixing $z_{n} \rightarrow \infty$. The next simplest case is represented by tadpole graphs, as depicted in Fig. 2(a), in which a single labeled line connects to the subcycle. One can reduce them to a sum of labeled trees by recursively using the IBP relation [16]

$$
\begin{aligned}
\operatorname{PT}(12 \cdots m) \stackrel{\mathrm{IBP}}{\cong} & \left(\sum_{\ell=2}^{m} \sum_{j=m+1}^{n-1} \sum_{\rho \in X \amalg Y^{T}} \frac{(-1)^{|Y|}}{1-s_{12 \cdots m}}\right. \\
& \left.\times \frac{s_{\ell j}}{z_{1 \rho_{1}} z_{\rho_{1} \rho_{2}} \cdots z_{\rho|\rho|} z_{\ell j}}\right),
\end{aligned}
$$

where $X$ and $Y$ are obtained by matching $(1,2 \cdots m)=$ $(1, X, \ell, Y)$. This relation holds when $\mathrm{PT}(12 \cdots m)$ is multiplied by a function independent of $z_{2}$ to $z_{m}$. The total derivative of $z_{1}$ is not used to derive this relation, and we identify it as the node shared by the subcycle and the tail. For example, Fig. 2(a) can be processed as

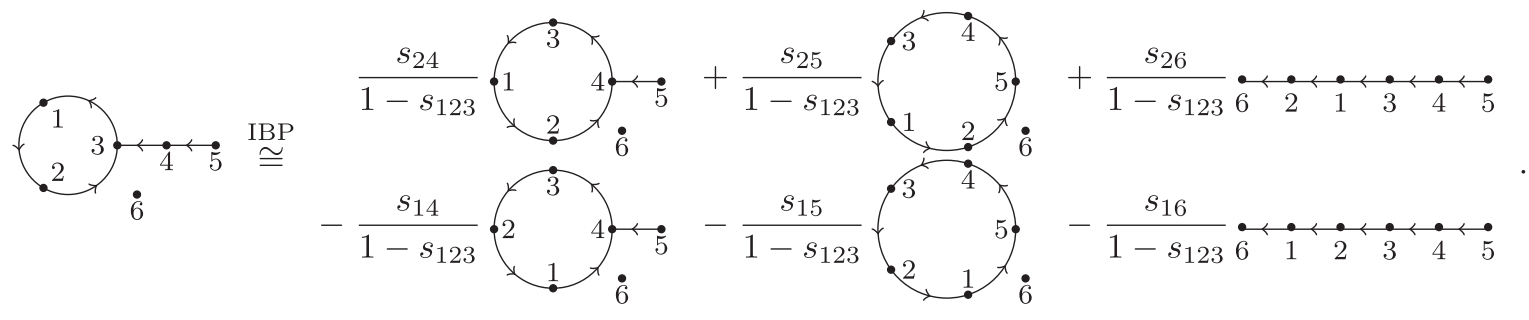

Notice that the tail always gets shortened, such that we can turn tadpole graphs into a sum of labeled trees by repeating the IBP relation (6).

By connecting more labeled lines to a tadpole, we get multibranch graphs, as shown in Fig. 2(b). If we remove a subgraph including part of the subcycle, the rest of the graph turns into a labeled tree, which can be written as a sum of labeled lines through algebraic operations [55]. For example, we can view the chain $\{4,1,2,3,5\}$ in Fig. 2(b) as a labeled tree rooted on 1 . Then partial fraction identities lead to

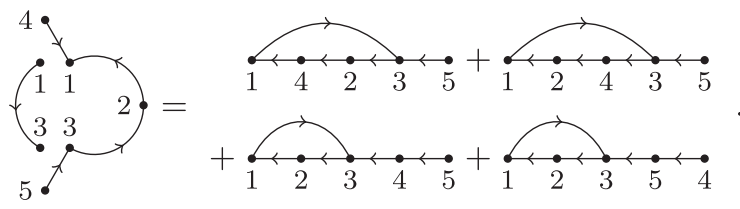


In this way, we can turn all the multibranch graphs into tadpoles and consequently labeled trees via IBP.

The most generic graph with no $z_{i j}$ in the numerator or connected subcycles has the form of Fig. 2(c), consisting of a product of tadpole and multibranch graphs, and a single labeled tree [56]. Following the above prescription, we can turn a tadpole or multibranch subgraph into labeled trees planted on the other connected components, reducing the number of subcycles by one. This algorithm allows us to recursively eliminate all the subcycles, and the final result contains only labeled trees; thus the function $\mathcal{I}_{n}^{\prime}$ we obtain is logarithmic.

As we will see shortly, the above discussion applies to open bosonic and heterotic strings. We defer the treatment of generic rational functions involving numerators and connected subcycles to [57].

String double copy: Now we prove that $\mathcal{M}_{n}^{\mathrm{FT}}$ given by the $\mathcal{I}_{n}^{\prime}$ obtained above is indeed the one in the double copy $\mathcal{M}_{n}^{\text {string }}=\mathcal{M}_{n}^{\mathrm{FT}} \otimes Z_{n}$. As a logarithmic function, we can write $\mathcal{I}_{n}^{\prime}=\sum_{\pi} N_{\pi} \mathrm{PT}(\pi)$, but the coefficients $N_{\pi}$ are not unique since the PT factors are not linearly independent. As shown for open-strings [9,17] and CHY integrals [26], they satisfy BCJ relations by IBP and SE equivalence moves, respectively. Consequently, any of them can be expressed in terms of the $(n-3)$ ! dimensional minimal basis

$$
\mathrm{PT}(\pi) \stackrel{\mathrm{IBP} / \mathrm{SE}}{\cong} \sum_{\beta \in S_{n-3}}\left(\sum_{\alpha \in S_{n-3}} m(\pi \mid \alpha) S[\alpha \mid \beta]\right) \mathrm{PT}(\beta) .
$$

By using Eqs. (2) and (3), we have

$$
\mathcal{M}_{n}^{\text {string }}(\rho)=\sum_{\alpha, \beta \in S_{n-3}}\left(\sum_{\pi} N_{\pi} m(\pi \mid \alpha)\right) S[\alpha \mid \beta] Z_{\rho}(\beta),
$$

for any $\rho \in S_{n} / Z_{n}$. The sum in the large parentheses is a field-theory amplitude defined by CHY formula: recalling that $m(\pi \mid \alpha)=\int d \mu_{n}^{\mathrm{CHY}} \mathrm{PT}(\pi) \mathrm{PT}(\alpha)$, thus we have

$$
\sum_{\pi} N_{\pi} m(\pi \mid \alpha)=\int d \mu_{n}^{\mathrm{CHY}} \underbrace{\sum_{\pi} N_{\pi} \mathrm{PT}(\pi)}_{\mathcal{I}_{n}^{\prime}} \mathrm{PT}(\alpha),
$$

which gives exactly $\mathcal{M}_{n}^{\mathrm{FT}}(\alpha)$ as in Eq. (4). Note that Eq. (9) makes it manifest that $\mathcal{M}_{n}^{\mathrm{FT}}(\rho)$ satisfies BCJ relations, which is necessary for a consistent KLT double copy. One can also define $\mathcal{M}_{n}^{\mathrm{FT}}$ by the LHS, which can be rewritten in a form that respects color or kinematics duality with BCJ numerators given by linear combinations of $N_{\pi}$ 's. However, we find it extremely useful to turn to $\mathrm{CHY}$ formula on the RHS: from our explicit result $\mathcal{I}_{n}^{\prime}$, we can use SE to simplify it to some $\mathcal{I}_{n}^{\prime \prime}$, for which it is possible to obtain a closed form.

One can apply the IBP reduction to type-I correlators $\mathcal{I}_{n}=\mathcal{K}_{n}(z)$ and obtain logarithmic functions, where the coefficients $N_{\pi}$ give the BCJ numerators for SYM [10]. For gluons, the standard CHY half-integrand $\mathcal{I}_{n}^{\prime \prime}=\operatorname{Pf}^{\prime} \Psi_{n}$ can also be reduced via $\mathrm{SE}$ to $\mathcal{I}_{n}^{\prime}$, giving equivalent $\mathrm{BCJ}$ numerators [67]. Remarkably, these BCJ numerators are free of any tachyon poles (though they appear in intermediate steps as in above examples), and unlike $\mathcal{I}_{n}$, both $\mathcal{I}_{n}^{\prime}$ and $\mathcal{I}_{n}^{\prime \prime}$ are homogenous in $\alpha^{\prime}$, all of which are of course guaranteed by the worldsheet supersymmetry. Next we will turn to bosonic or heterotic strings, where BCJ numerators and $\mathcal{I}_{n}^{\prime}$ contain tachyon poles and nontrivial $\alpha^{\prime}$ dependence. We will see that our two-step method again gives a remarkably simple formula for $\mathcal{I}_{n}^{\prime \prime}$.

New formulas for bosonic and heterotic strings. - Let us first review correlators for (compactified) open bosonic strings and heterotic strings. Since the former is identical to the holomorphic part of the latter, we will focus on the heterotic-string case and everything carries over to the open-string case. By a slight abuse of notation, we denote closed-string measure as $\left|d \mu_{n}^{\text {string }}\right|^{2}:=$ $\left\{\left[d^{n} z d^{n} \bar{z} \mathrm{KN}\right] /[\operatorname{volSL}(2, \mathbb{C})]\right\}$, and the single-trace tree amplitude of $m$ gravitons and $r=n-m$ gluons is given by Ref. [16]

$$
\mathcal{M}_{m, r}^{\text {het }}(\rho)=\int \mid d \mu_{n}^{\text {string }}{ }^{2} \mathrm{PT}(\rho) R_{m}(z) \mathcal{K}_{n}(\bar{z})
$$

where $\rho \in S_{r} / Z_{r}$ denotes the gluon color ordering. The holomorphic function $R_{m}$ contains half of the graviton vertex operator correlation function, taking the form

$$
R_{m}(z)=\sum_{(I)(J) \cdots(K) \in S_{m}} \mathcal{R}_{(I)} \mathcal{R}_{(J)} \cdots \mathcal{R}_{(K)},
$$

where we sum over permutations in $S_{m}$, each of which is written as a product of disjoint cycles. The cycle factor $\mathcal{R}_{(I)}$ is given by $\mathcal{R}_{(a)}=\sum_{b \neq a}\left(\epsilon_{a} \cdot k_{b} / z_{a b}\right):=C_{a}, \mathcal{R}_{(a b)}=$ $\left(\epsilon_{a} \cdot \epsilon_{b} / \alpha^{\prime} z_{a b}^{2}\right)$, and $\mathcal{R}_{(I)}=0$ for $|I|>2$. The antiholomorphic function $\mathcal{K}_{n}(\bar{z})$ is the type-I correlator that encodes vertex operators of gluons and the other half of the gravitons.

For the single-trace integrand, we can always gauge fix the gluon $z_{n} \rightarrow \infty$ such that we only need to break the $z$ cycles in $R_{m}(z)$ by IBP. The one-graviton integrand is automatically in the logarithmic form since $R_{1}=C_{1}$ contains no $z$ cycles, i.e., $\mathcal{I}_{n}(1 ; \rho)=\mathcal{I}_{n}^{\prime}(1 ; \rho)=\operatorname{PT}(\rho) C_{1}$. For two gravitons, we have $R_{2}(z)=C_{1} C_{2}+\left(\epsilon_{1} \cdot \epsilon_{2} / \alpha^{\prime} z_{12}^{2}\right)$, which has only one $z$-cycle $1 / z_{12}^{2}$. We can break it by the IBP

$$
\frac{1}{z_{12} z_{21}}(\cdots) \stackrel{\operatorname{IBP}}{\cong} \sum_{i=3}^{n-1} \frac{s_{2 i}}{1-s_{12}} \frac{1}{z_{12} z_{2 i}}(\cdots),
$$

which holds when $(\cdots)$ does not involve $z_{2}$. This leads to the logarithmic function 


$$
\begin{aligned}
\mathcal{I}_{n}^{\prime}(12 ; \rho)= & \left(\sum_{i=3}^{n-1} \frac{\epsilon_{1} \cdot k_{i} z_{i n}}{z_{1 i} z_{1 n}} \sum_{j=3}^{n-1} \frac{\epsilon_{2} \cdot k_{j} z_{j n}}{z_{2 j} z_{2 n}}\right. \\
& \left.+\frac{\alpha^{\prime} \epsilon_{1} \cdot k_{2} \epsilon_{2} \cdot k_{1}-\epsilon_{1} \cdot \epsilon_{2}}{\alpha^{\prime}\left(1-s_{12}\right) z_{12}} \sum_{i=3}^{n-1} \frac{s_{2 i} z_{i n}}{z_{2 i} z_{1 n}}\right) \operatorname{PT}(\rho),
\end{aligned}
$$

with the SL(2) covariance restored. As another example, we present the IBP of the three-graviton integrand in Ref. [57]. Similar calculation can also be found in Ref. [16] for a slightly different purpose. Although the complexity of $\mathcal{I}_{n}^{\prime}$ grows quickly with more gravitons, it is still well controlled under our algorithm. Moreover, as the second step, we can greatly simplify $\mathcal{I}_{n}^{\prime}$ by using SE and obtain a closed-form $\mathcal{I}_{n}^{\prime \prime}$ for the most generic single-trace sector.

Similar treatment can in principle be applied to multitrace and pure graviton sectors. We give several such examples in Ref. [57].

The single-trace integrand: Now we present the singletrace $\mathrm{CHY}$ half-integrand $\mathcal{I}_{n}^{\prime \prime}$, resulted from our two-step reduction of string integrand (10)

$$
\mathcal{I}_{n}^{\prime \prime}(12 \cdots m ; \rho)=\operatorname{PT}(\rho) \mathcal{P}_{m},
$$

where $\mathcal{P}_{m}$ is given by the cycle expansion

$$
\mathcal{P}_{m}=\sum_{(I)(J) \cdots(K) \in S_{m}} \Psi_{(I)} \Psi_{(J)} \cdots \Psi_{(K)} .
$$

For length one and two cycles, we have $\Psi_{(a)}=C_{a}$ and $\Psi_{(a b)}=-T_{a b} \mathrm{PT}(a b)$, where $T_{a b}$ is given by $T_{a b}:=$ $[\operatorname{tr}(a b)] /\left[\left(1-s_{a b}\right)\right]:=\frac{1}{2}\left\{\left[\operatorname{tr}\left(f_{a} f_{b}\right)\right] /\left[\left(1-s_{a b}\right)\right]\right\}$, and $f_{a}^{\mu \nu}=$ $k_{a}^{\mu} \epsilon_{a}^{\nu}-k_{a}^{\nu} \epsilon_{a}^{\mu}$ is the linearized field strength. For longer cycles $(|I| \geq 3)$, we have $\Psi_{(I)}=-T_{I} \mathrm{PT}(I) / 2$, where $T_{I}$ is recursively generated from $T_{a b}$ through

$$
T_{I}=\frac{1}{1-s_{I}}\left(\operatorname{tr}(I)+\sum_{\mathrm{CP}} G_{i_{2}}^{I_{1}} G_{i_{3}}^{I_{2}} \cdots G_{i_{1}}^{I_{p}}\right),
$$

with $\operatorname{tr}(I):=\operatorname{tr}\left(f_{a_{1}} f_{a_{2}} \cdots f_{a_{|I|}}\right)$ for $|I| \geq 3$. In this equation, the summation is over all cyclic partitions $(C P)$ of a cycle $I$ into words, $\left\{I_{1}, I_{2}, \ldots, I_{p}\right\}$ where the length of each word $I_{\ell}$ is at least two; $i_{\ell}$ denotes the first element of $I_{\ell}$, which is the label in $I$ that succeeds the word $I_{\ell-1}$. For example, for $I=(1,2,3,4)$, we have four CPs with only one word, $1234,2341,3412,4123$, and two more with two words, $\{12,34\}$ and $\{23,41\}$; the sum on the RHS of Eq. (16) thus include terms like $G_{1}^{1234}$, and $G_{3}^{12} G_{1}^{34}$, etc. Given a word $A=a_{1} a_{2} \cdots a_{s}$ and the next label $b$, the function $G$ is defined as

$$
G_{b}^{A}=\sum_{q=2}^{s-\delta_{a_{1} b}} T_{a_{1} a_{2}\left[a_{3}\left[\cdots\left[a_{q-1} a_{q}\right] \cdots\right]\right]} V_{a_{q+1} \cdots a_{r}}^{a_{q} b},
$$

where $V_{a_{q+1} \cdots a_{r}}^{a_{q} b}=\alpha^{\prime} k_{a_{q}} \cdot f_{a_{q+1}} \cdots f_{a_{r}} \cdot k_{b}$, and in particular $V^{a b}=s_{a b}$. The bracket $[i j]$ stands for the index antisymmetrization, for example, $T_{a b[c d]}:=T_{a b c d}-T_{a b d c}$. The summation range $s-\delta_{a_{1} b}$ ensures that the right-hand side of Eq. (16) only contains those $T$ s with shorter cycles, since $b$ agrees with $a_{1}$ only when $A=I$. In Ref. [57], we give examples of $T_{I}$ up to five gluons.

Note that the closed formula for $\mathcal{I}_{n}^{\prime \prime}$ is still a conjecture, since it is generalized from explicit calculations at low points. However, as will be discussed in great detail in Ref. [68], the formula has passed various nontrivial consistency checks. On the support of SE, we have checked numerically up to 11 points that Eq. (14) agrees with the logarithmic form $\mathcal{I}_{n}^{\prime}$ resulted from the IBP of the string integrand (10). We have also checked up to eight points that the gauge amplitude produced by Eq. (14) agrees with that calculated from the Feynman diagrams of $(D F)^{2}+\mathrm{YM}+\phi^{3}$ with massless external particles. Further consistency checks are provided in Ref. [57].

Conclusion and discussion.-In this Letter, we have presented an IBP reduction method for correlators of any massless string amplitude, $\mathcal{I}_{n}$, to a class of logarithmic functions, $\mathcal{I}_{n}^{\prime}$. The latter can be used as CHY half-integrand (with the other half given by PT) to define field-theory amplitudes, and their double copy with disk or sphere integrals gives the original string amplitude. Although $\mathcal{I}_{n}^{\prime}$ generally takes a complicated form, one can use SE to simplify it and even get a closed form to all multiplicities. We have demonstrated this two-step method for open (compactified) bosonic or heterotic strings and obtained remarkably simple formulas for single-trace and certain multitrace sectors of $(D F)^{2}+\mathrm{YM}+\phi^{3}$ theory. It can bring new insight into this theory, as well as conformal gravity which is obtained from a double copy with YM $[23,69]$.

Our discussion is completely generic since it only requires $\mathcal{I}_{n}$ to be a rational function with correct $\operatorname{SL}(2)$ weight. It can thus be applied beyond those well-studied conventional string theories, for example, to the dual model proposed in Ref. [70]. An important open question concerns the application of IBP reduction to string correlators with massive states, and it would be fascinating to see if similar structures exist there.

On the other hand, the CHY half-integrands obtained in this Letter contain explicit $\alpha^{\prime}$ dependence. It will be interesting to understand how they can emerge from certain model of ambitwistor string [32,36,37]. Some attempts have been made along this direction and give the correct three-point results [40]. Last, but not least, it is highly desirable to extend certain aspects of our construction to loop-level string amplitudes or correlators, and see how they are related to field-theory amplitudes or ambitwistor string correlators.

It is our pleasure to thank Thales Azevedo, Henrik Johansson, Sebastian Mizera, and Oliver Schlotterer for 
inspiring discussions and helpful comments on our draft. S. H.'s research is supported in part by the Thousand Young Talents program, the Key Research Program of Frontier Sciences of CAS under Grant No. QYZDB-SSW-SYS014, and Peng Huanwu center under Grant No. 11747601. F. T. is supported by the Knut and Alice Wallenberg Foundation under Grant No. KAW 2013.0235 and the Ragnar Söderberg Foundation under Grant No. S1/16.

*songhe@itp.ac.cn

${ }^{\dagger}$ fei.teng@physics.uu.se *yongzhang@itp.ac.cn

[1] N. Arkani-Hamed, J. L. Bourjaily, F. Cachazo, A. B. Goncharov, A. Postnikov, and J. Trnka, Grassmannian Geometry of Scattering Amplitudes (Cambridge University Press, Cambridge, United Kingdom, 2016).

[2] J. M. Henn and J. C. Plefka, Lect. Notes Phys. 883, 1 (2014).

[3] H. Elvang and Y.-t. Huang, Scattering Amplitudes in Gauge Theory and Gravity (Cambridge University Press, Cambridge, United Kingdom, 2015).

[4] H. Kawai, D. C. Lewellen, and S. H. H. Tye, Nucl. Phys. B269, 1 (1986).

[5] Z. Bern, L. J. Dixon, M. Perelstein, and J. S. Rozowsky, Nucl. Phys. B546, 423 (1999).

[6] Z. Bern, J. J. M. Carrasco, and H. Johansson, Phys. Rev. D 78, 085011 (2008).

[7] Z. Bern, J. J. M. Carrasco, and H. Johansson, Phys. Rev. Lett. 105, 061602 (2010).

[8] C. R. Mafra, O. Schlotterer, and S. Stieberger, J. High Energy Phys. 07 (2011) 092.

[9] C. R. Mafra, O. Schlotterer, and S. Stieberger, Nucl. Phys. B873, 419 (2013).

[10] C. R. Mafra, O. Schlotterer, and S. Stieberger, Nucl. Phys. B873, 461 (2013).

[11] C. R. Mafra and O. Schlotterer, Fortschr. Phys. 63, 105 (2015).

[12] S. He, R. Monteiro, and O. Schlotterer, J. High Energy Phys. 01 (2016) 171.

[13] N. E. J. Bjerrum-Bohr, P. H. Damgaard, and P. Vanhove, Phys. Rev. Lett. 103, 161602 (2009).

[14] S. Stieberger, arXiv:0907.2211.

[15] S. Stieberger and T. R. Taylor, Nucl. Phys. B913, 151 (2016).

[16] O. Schlotterer, J. High Energy Phys. 11 (2016) 074.

[17] J. Broedel, O. Schlotterer, and S. Stieberger, Fortschr. Phys. 61, 812 (2013).

[18] J. J. M. Carrasco, C. R. Mafra, and O. Schlotterer, J. High Energy Phys. 06 (2017) 093.

[19] C. R. Mafra and O. Schlotterer, J. High Energy Phys. 01 (2017) 031.

[20] J. J. M. Carrasco, C. R. Mafra, and O. Schlotterer, J. High Energy Phys. 08 (2017) 135.

[21] Y.-t. Huang, O. Schlotterer, and C. Wen, J. High Energy Phys. 09 (2016) 155.

[22] T. Azevedo, M. Chiodaroli, H. Johansson, and O. Schlotterer, J. High Energy Phys. 10 (2018) 012.

[23] H. Johansson and J. Nohle, arXiv:1707.02965.
[24] O. Schlotterer and S. Stieberger, J. Phys. A 46, 475401 (2013).

[25] F. Cachazo, S. He, and E. Y. Yuan, Phys. Rev. Lett. 113, 171601 (2014).

[26] F. Cachazo, S. He, and E. Y. Yuan, J. High Energy Phys. 07 (2014) 033.

[27] F. Cachazo, S. He, and E. Y. Yuan, Phys. Rev. D 90, 065001 (2014).

[28] T. Adamo, E. Casali, and D. Skinner, J. High Energy Phys. 04 (2014) 104.

[29] Y. Geyer, L. Mason, R. Monteiro, and P. Tourkine, Phys. Rev. Lett. 115, 121603 (2015).

[30] F. Cachazo, S. He, and E. Y. Yuan, J. High Energy Phys. 08 (2016) 008.

[31] Y. Geyer, L. Mason, R. Monteiro, and P. Tourkine, Phys. Rev. D 94, 125029 (2016).

[32] Y. Geyer and R. Monteiro, J. High Energy Phys. 11 (2018) 008.

[33] F. Cachazo, S. He, and E. Y. Yuan, J. High Energy Phys. 07 (2015) 149.

[34] S. He and O. Schlotterer, Phys. Rev. Lett. 118, 161601 (2017).

[35] S. He, O. Schlotterer, and Y. Zhang, Nucl. Phys. B930, 328 (2018).

[36] L. Mason and D. Skinner, J. High Energy Phys. 07 (2014) 048.

[37] E. Casali, Y. Geyer, L. Mason, R. Monteiro, and K. A. Roehrig, J. High Energy Phys. 11 (2015) 038.

[38] W. Siegel, arXiv:1512.02569.

[39] E. Casali and P. Tourkine, J. High Energy Phys. 11 (2016) 036.

[40] T. Azevedo and R. L. Jusinskas, J. High Energy Phys. 10 (2017) 216.

[41] S. Mizera, Phys. Rev. Lett. 120, 141602 (2018).

[42] N. E. J. Bjerrum-Bohr, P. H. Damgaard, P. Tourkine, and P. Vanhove, Phys. Rev. D 90, 106002 (2014).

[43] N. E. J. Bjerrum-Bohr, P. H. Damgaard, B. Feng, and T. Sondergaard, J. High Energy Phys. 09 (2010) 067.

[44] S. Mizera, J. High Energy Phys. 08 (2017) 097.

[45] N. Arkani-Hamed, Y. Bai, and T. Lam, J. High Energy Phys. 11 (2017) 039.

[46] N. Arkani-Hamed, Y. Bai, S. He, and G. Yan, J. High Energy Phys. 05 (2018) 096.

[47] See Sebastian Mizera's talk at Amplitude 2018.

[48] We refer the readers to the original paper [25] for the definition of the $2 n \times 2 n$ matrix $\Psi_{n}$ and more details on $\mathrm{CHY}$ formulas.

[49] S. Stieberger, J. Phys. A 47, 155401 (2014).

[50] S. Stieberger and T. R. Taylor, Nucl. Phys. B881, 269 (2014).

[51] O. Schlotterer and O. Schnetz, J. Phys. A 52, 045401 (2019)

[52] F. Brown and C. Dupont, arXiv:1810.07682.

[53] O. Schnetz, Commun. Num. Theor. Phys. 08, 589 (2014).

[54] F. Brown, SIGMA 2, e25 (2014).

[55] X. Gao, S. He, and Y. Zhang, J. High Energy Phys. 11 (2017) 144.

[56] If there are more than one labeled trees, there must also be connected subcycles as a result of the SL(2) weight constraint. 
[57] See Supplemental Material at http://link.aps.org/ supplemental/10.1103/PhysRevLett.122.211603 for examples and a few technical details, which includes Refs. [58-66].

[58] S. Weinberg, Phys. Rev. 140, B516 (1965).

[59] M. Chiodaroli, M. Günaydin, H. Johansson, and R. Roiban, J. High Energy Phys. 01 (2015) 081.

[60] F. Cachazo, S. He, and E. Y. Yuan, J. High Energy Phys. 01 (2015) 121.

[61] C. S. Lam and Y.-P. Yao, Phys. Rev. D 93, 105008 (2016).

[62] C. Cardona, B. Feng, H. Gomez, and R. Huang, J. High Energy Phys. 09 (2016) 133.

[63] N. E. J. Bjerrum-Bohr, J. L. Bourjaily, P. H. Damgaard, and B. Feng, J. High Energy Phys. 09 (2016) 094.
[64] S. He and Y. Zhang, J. High Energy Phys. 02 (2017) 019.

[65] R. Huang, Y.-J. Du, and B. Feng, J. High Energy Phys. 06 (2017) 133.

[66] M. Chiodaroli, M. Günaydin, H. Johansson, and R. Roiban, J. High Energy Phys. 07 (2017) 002.

[67] Y.-J. Du and F. Teng, J. High Energy Phys. 04 (2017) 033.

[68] S. He, F. Teng, and Y. Zhang (in preparation).

[69] H. Johansson, G. Mogull, and F. Teng, J. High Energy Phys. 09 (2018) 080.

[70] C. Baadsgaard, N. E. J. Bjerrum-Bohr, J. L. Bourjaily, and P. H. Damgaard, J. High Energy Phys. 12 (2016) 019. 